\title{
Reversing priming while maintaining interference
}

\author{
ANTHONY BEECH and KIRSTEN AGAR \\ University of Oxford, Oxford, England \\ and \\ GORDON C. BAYLIS \\ University of California, San Diego, La Jolla, California
}

\begin{abstract}
The amount of interference and priming is examined in a Stroop color-naming task, in which distractors were either color words (conventional Stroop) or nonwords comprising the first two letters of the color words (color pseudowords). It was found that both color words and color pseudowords produced an interference effect, and that the amount of interference by these two types of stimuli was highly correlated across subjects. It was also found that color words led to a reaction time cost when distractor and subsequent target were the same, compared to trials on which no such relation existed. This was an instance of negative priming (Tipper, 1985). Conversely, color pseudowords led to positive priming and reaction time saving, under the same conditions. These data suggest that interference is related to the initial structural elements of the words, and that it may occur regardless of whether or not words can be fully analyzed, whereas priming effects may depend on whether it is possible to analyze the stimuli on a semantic level.
\end{abstract}

Selective attention is a process responsible for minimizing the interference effects of unwanted material on target processing. Broadly speaking, this process has been argued to occur relatively early, in the perceptual system (Broadbent, 1958) or at the stage of response (Allport, 1980; Deutsch \& Deutsch, 1963; van der Heijden, Hagenaar, \& Bloem, 1984). According to early selection theories, only low-level features are processed preattentively, and attentional effort is necessary for these to become integrated into a perceptual representation. Therefore, nontarget information will receive minimal processing. According to late selection theories, on the other hand, selection occurs only after all stimuli are fully analyzed, and thus there is no limit to the amount of information that can be processed simultaneously prior to the response stage.

Although most theories of selection assume that unattended information is lost by passive decay, it has been argued that active inhibition of irrelevant stimuli is an important process in selective attention (Deutsch \& Deutsch, 1963; Neill, 1977; Norman \& Shallice, 1985; Tipper, 1985). This has been demonstrated with the Stroop (1935) effect: When the color word that has previously been ignored on trial $n$ becomes the ink color name to be named on trial $n+1$, a reaction time cost is incurred (Beech \& Claridge, 1987; Dalrymple-Alford \& Budayr, 1966). This effect has also been demonstrated with pictures and letters (Tipper, 1985; Tipper \& Cranston, 1985), and the more

Correspondence may be addressed to Gordon C. Baylis, Department of Psychology C-009, University of California, San Diego, La Jolla, CA 92093. general finding of the suppression of unattended information has been termed negative priming.

The experiment reported here was designed to investigate the relation between concurrent effects (i.e., within a given trial) of nontarget stimuli and the priming effects arising from them on subsequent trials. Intratrial interference is often seen as a measure of the inefficiency of passive filtering - that is, of the degree to which nontarget information has been processed in the absence of a requirement to do so. Repetition priming from nontargets on trial $n$ to the target on trial $n+1$ could also be seen in a similar way-that is, activation of the representation of the nontarget on trial $n$ was due to nontarget "breakthrough," and such activation led to a saving on trial $n+1$. Conversely, negative priming at trial $n+1$ would be seen by Tipper (1985) as a biproduct of efficient selection on trial $n$, resulting from a process of active inhibition characteristic of efficient selection.

This experiment was conducted to examine the intertrial priming effects of meaningful and meaningless nontarget stimuli that (despite considerable differences) lead to the same amount of intertrial interference.

\section{METHOD}

\section{Subjects}

Thirty members of the Oxford Subject Panel took part in the experiment: 13 male, 17 female. Their ages ranged from 21 to $48(M=32.5$, $S D=7.9$ ) years. All had normal color vision and normal or correctedto-normal visual acuity.

\section{Stimuli}

The stimulus "ink" colors were red, pink, green, and blue, in which the (distractor) words were written. The experimental distractors were 
the color words RED, PINK, GREEN, and BLUE, written in uppercase teletext characters. The pseudodistractors were matched in length to, and made up of the first two letters of, the corresponding color word followed by frequency-matched vowels and consonants-for example, REL, PIRF, GROIT, and BLOR. In the condition in which no distractors were present (ND; see below), the target color was displayed as a row of Xs, randomly of 3, 4, or 5 characters in length.

\section{Design}

The experiment consisted of a within-subjects design, whereby 40 lists were presented in four blocks of 10 , with each list comprising nine display trials. All trials in a given list shared the same condition, which could be one of the five listed below. In each of the four blocks, each of the conditions occurred twice in pseudorandom order. The reaction times (RTs) and error rates were taken from List Positions 2-9, resulting in a total of 64 trials per cell.

Three conditions were determined by intratrial relations of target to distractor:

Neutral distractor (ND)-A row of Xs in successively varied colors was used instead of Stroop words. Thus the target ("ink") occurred in the absence of a distractor.

Color-word distractor (CD)-A color word was presented in the target hue. The "ink" color and distractor were unrelated. There was also no relation between the word on trial $n$ and the "ink" on trial $n+1$.

Pseudoword distractor (PD)-Nonsense words were presented in the target "ink." The color word that corresponded to the pseudoword was also unrelated to the "ink" on trial $n$ and $n+1$ (as in condition CD).

The pseudoword distractors were used to assess the (intratrial) interference effects of color words and pseudowords in the following way: Interference due to color words was calculated by subtracting the baseline (ND) from the RT to displays in which color-word distractors were present $(C D)$. In an analogous way, the priming effect of pseudowords was measured as the difference PD-ND. A further two conditions were defined by intertrial relations of stimuli:

Ignored repetition of color word (CIR)-A Stroop color name predicted the hue of the following Stroop word.

Ignored repetition of pseudoword (PIR)-Nonsense words were presented, the first two letters of which predicted the first two letters of the hue of the following word.

The amount of (intertrial) priming due to color words was calculated by subtracting the mean RT for the CD condition from the mean RT for the CIR condition, and the amount of pseudoword priming by subtracting the mean RT for the PD condition from that of the PIR condition. In both cases, a negative value indicated negative priming and a positive value indicated facilitation.

\section{Apparatus}

The stimuli were generated on an ACORN BBC microcomputer (Model $\mathrm{B}+$ ) with 32K available RAM, and presented on a Microvitec Cub 895 monitor. The stimuli subtended $1.0^{\circ}$ vertically and $2.0^{\circ}-3.5^{\circ}$ horizontally, and were presented on fixation. The letters had a mean luminance of approximately $3.5 \mathrm{fL}$ and were presented on a black field of approximately $0.25 \mathrm{fL}$. A TTL voice key interfaced to the computer enabled verbal RTs to be measured. Timing was measured to a resolution of $1 \mathrm{msec}$, using low-level access to the internal clock of the computer.

\section{Procedure}

The subjects were instructed to name the "ink" color of each stimulus, to ignore any other information displayed, and to respond as quickly as possible. The sequence of events was as follows: Each subject pressed a button to trigger each list; then, after a gap of $500 \mathrm{msec}$, a fixation cross appeared for $100 \mathrm{msec}$, followed by a stimulus for $500 \mathrm{msec}$. This was followed by a pattern mask until the subject triggered the voice key, when the next fixation cross appeared, and so on. RTs to every display in the list were taken, although only Positions 2-9 were used in the subsequent analysis (no priming could take place on the first position of the list). At the end of each list, a display informed the subject of the total RT to it and the number of errors made in naming the items in the list. Five practice lists were given to each subject before the experimental trials began.

\section{RESULTS}

The mean response latencies to the five conditions are shown in Figure 1. A Freidman one-way analysis of variance (Wilkinson, 1988) showed a highly significant effect of condition $[F(4,116)=78.7, p<.0001]$. To analyze the data further, Wilcoxon post hoc tests were used to assess the significance of specific contrasts (in all cases the $Z$ score of the computed Wilcoxon $T$ is quoted along with two-tailed probabilities, for conservatism). The expected Stroop effect and negative priming effect were both highly significant $(Z=4.8, P<.001$, and $Z=3.2$, $P<.001$, respectively). Similar tests showed that there was significant interference by pseudowords (PD vs. ND: $Z=4.7, P<.001$ ), as well as positive priming by pseudowords (PIR vs. PD; $Z=3.9, P<.001$ ).

A similar analysis of variance carried out on the errors showed that there was a highly significant effect of condition $[F(4,116)=21.3, p<.001]$. It can be seen from Figure 1 that in all cases, the pattern of errors closely followed that of the reaction times. In addition, post hoc Wilcoxon tests showed that the Stroop interference (CD-ND) and interference by pseudowords (PD vs. ND) were both significant $(Z=3.4, P<.001$, and $Z=3.7$, $P<.001$, respectively). However, both the negative priming effect of color words and the positive priming effect of pseudowords failed to reach significance in the two-tailed model used $(Z=1.8, P<.08$, and $Z=1.0$, $P<.18$, respectively), although they approach significance in a reasonable, though less conservative onetailed test.

The amount of interference due to color words was almost the same as that due to the pseudowords. In order

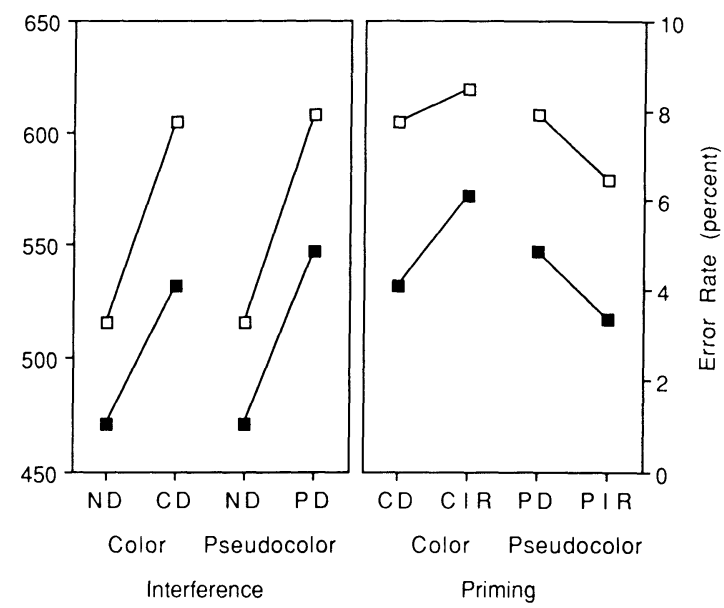

Figure 1. The mean reaction times (open symbols) and mean error rates (filled symbols) for the five experimental conditions. On the left are shown the intratrial interference effects of color words and pseudowords; on the right are shown the intertrial effects due to ignored color words and pseudowords. Note that intratrial effects share the same baseline condition (ND), whereas intertrial effects use the appropriate distractor condition (CD or PD) as a baseline. Abbreviations: ND: no distractor; CD: unrelated distractor; PD: unrelated pseudodistractor; CIR: ignored repetition; PIR: ignored pseudorepetition. 


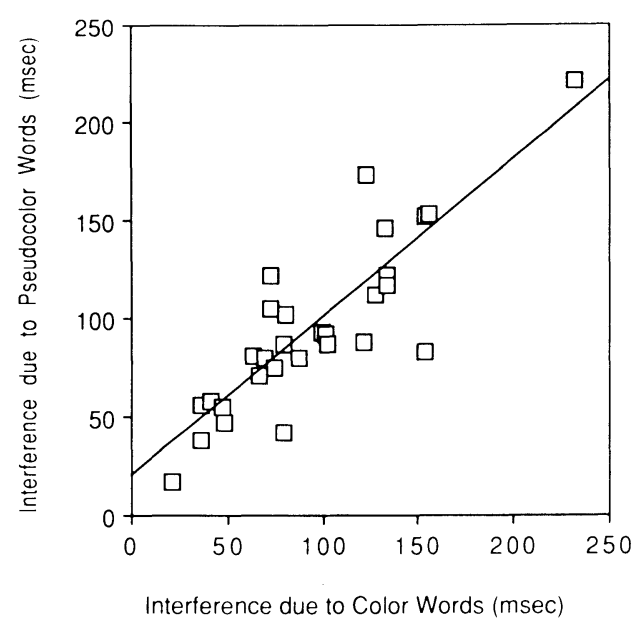

Figure 2. The mean interference (in milliseconds) due to the color pseudoword distractors plotted against the interference due to the color-word distractors. Each point represents the data for 1 subject. The regression line is also shown.

to understand the relation of these two measures of interference further, the values of each measure were plotted against each other for the 30 subjects. In Figure 2, the interference due to color words (CD-ND) is plotted against interference due to pseudowords (PD-ND). Each point represents the pair of data for a single subject (two data points overlie each other). It can be seen that the two measures are highly correlated $(R=.85, P<.001)$. Moreover, the slope of the regression line (shown on this figure) is close to unity, which of course need not be the case, however close to unity the correlation coefficient may be.

\section{DISCUSSION}

This experiment has shown that color words and pseudowords sharing the same first two letters as color words can both act as distractors in a Stroop color-naming task, a result similar to the findings of Singer, Lappin, and Moore (1975). The amount of interference due to the two different types of distractors was almost the same for a given subject. This suggests that the interference caused by these two types of distractors reflects the same mechanism and is largely due to featural elements of the initial two letters of the stimuli.

However, the priming effects found suggest that color words and pseudowords receive very different processing. Significant negative priming was observed when a color-word distractor was the same as the next target; significant positive priming was obtained from the pseudowords that had the corresponding relation. Tipper (1985; Tipper, Brehaut, \&
MacQueen, 1988) has suggested that inhibition occurs at the stage of response after categorical levels of representation have been achieved. In Condition CIR, when true color words were present, the distractor representation was inhibited, resulting in an RT cost on the next trial. In the analogous pseudoword condition (PIR) there was no competition between representations, since a pseudoword does not have a meaning and thus cannot have an associated response. However, the features of the pseudoword that are common to the color word (i.e., the first two letters) were available to (positively) prime the subsequent target.

Overall, the results suggest that, although there is interference at the level of features, this is not the stage at which selection finally takes place. Final selection occurs at a postcategorical level of representation, on which active inhibition of meaningful distractors plays a role.

\section{REFERENCES}

ALLPORT, D. A. (1980). Attention and performance. In G. Claxton (Ed.), Cognitive psychology: New directions (pp. 112-153). London: Routledge and Keegan Paul.

BEECH, A., \& ClARIDGe, G. (1987). Individual differences in negative priming. British Journal of Psychology, 78, 349-356.

BROADBENT, D. E. (1958). Perception and communication. London: Pergamon.

Dalrymple-Alford, E. C., \& BudAyr, B. (1966). Examination of some aspects of the Stroop color-word test. Perceptual \& Motor Skills, 23 , 1211-1214.

Deutsch, J. A., \& Deutsch, D. (1963). Attention: Some theoretical considerations. Psychological Review, 70, 80-90.

NEILL, W. T. (1977). Inhibition and facilitation processes in selective attention. Journal of Experimental Psychology: Human Perception \& Performance, 3, 444-450.

Norman, D. A., \& Shaluce, T. (1985). Attention to willed action: Willed and automatic control of behavior. In R. J. Davison, G. E. Schwartz, \& R. L. Shapiro (Eds.), Conscious and self regulation: Advances in research (Vol. 4). New York: Plenum.

Singer, M. H., LAPPIN, J. S., \& MoORE, L. P. (1975). The interference of various word parts on color naming in the Stroop test. Perception \& Psychophysics, 18, 191-193.

STROOP, J. R. (1935). Studies of interference in serial verbal reactions. Journal of Experimental Psychology, 18, 643-662.

TIPPER, S. P. (1985). The negative priming effect: Inhibitory priming by ignored objects. Quarterly Journal of Experimental Psychology, 37A, 591-611.

TipPer, S. P., \& Cranston, M. (1985). Selective attention and priming: Inhibitory and facilitatory effects of ignored primes. Quarterly Journal of Experimental Psychology, 37A, 591-611.

Tipper, S. P., MacQueen, G. M., \& Brehaut, J. C. (1988). Negative priming between response modalities: Evidence for the central locus of inhibition in selective attention. Perception \& Psychophysics, 43, 45-52.

van der Heijden, A. H. C., Hagenaar, R., \& Bloem, W. (1984). Two stages in postcategorical filtering and selection. Memory \& Cognition, 12, 458-469.

WILKINSON, L. (1988). SYSTAT: The system for statistics. Evanston, IL: Systat Inc.

(Manuscript received May 12, 1989.) 